\title{
Indice dei nomi
}

Abramio, Giovanni 95 e nn. 297, 300, 183

Acciaiuoli, Zanobi 151, 161, 166

Acindino, Gregorio 109, 240-242, 246

Acropolita, Giorgio n. 212

Aftonio n. 243

Agostino di Ippona (san) 114-115, 148, 150-151

Albumasar 95, 234

Alessandro di Afrodisia 86, 191-192

Allacci, Leone 166

Ammonio 182, 191

Anastasio Sinaita 109, 111-112 e n. 385, 115, $118,138,140$

Anonimo Crameri 204

Apostolio, Arsenio-Aristobulo 225

Appiano 71, 232

Aristofane 80,83 e n. $227,84-85$ e n. 235,86 , 105, 204-206

Aristofane Bizantino 201

Aristotele 8,16, n. $71,22,25,49,59,64,70$, $72,83,86$ e n. 244,87 e n. $248,91,95,97$ e n. $313,124,190-191,234$

Aristotele Ps. 250

Arsenio di Tiro 8 e n. 43, 20, n. 88, nn. 203, 341,172

Atanasio Alessandrino 100, 146

Atanasio Alessandrino Ps. 160, 164

Balsamone, Michele n. 388, 241

Barlaam Calabro 74, 88, 91, 126, 186, 188, 240, 255

Basilio di Cesarea, Magno 100, 112, 146, 212

Basilikos, Andronikos, copista n. 90

Baumgartner, Hieronymous 194-197

Becco, Giovanni 164-165

Berengario di Tours n. 331

Bessarione, Basilio, cardinale n. 270, 91, 240, 246, 248-249, 252-253, 255-257, 263

Blemmida, Niceforo 119, 163, 166

Briennio, Giuseppe 82

- Manuele 132,134-135

Bullote, Giorgio n. 55

Cabasila, Demetrio Canisce 131

Cabasila, Nicola 91, 149, 219-221, 223-225, 255

Caleca, Manuele 162, 166

Callisto, Andronico 201
Cantacuzeno, Giovanni VI, imperatore n. 55, $45,86,99$, n. $335,109,119,125-126,154$, 160-161

Caritone, copista 18 e n. 79

Carlo III di Borbone, re di Spagna 192

Carlo X di Borbone, re di Francia e Navarra 207

Carol de Montchal 234

Cassiodoro 82

Catrario, Giovanni n. 113

Cherobosco, Giorgio 85 e n. 237, 208, 237

Cicerone, Marco Tullio n. 275

Cidone, Demetrio n. 62, 29, 63, 67, n. 176, 79 , n. 210,99 e n. $322,100,109,115$ e n. 394 $119,126,128,138,150-151,153,155-158$, $160-164,166,245$

- Procoro n. 62, 29, 64, 66, 99 e n. 322, 109, 114 e n. 389, 115 e nn. 393-394, 126, 128, 140, 147-148, 151, 153, 158-159, 244

Ciparissiota, Giovanni 52, 56 e n. 148, 59, 61, 63, 65-67, 69 e n. $170,70,79,99$ e n. 321 , n. 335,103 e n. 346, n. 371,111 e n. 379 , 114-115, 121, 126, 142-143, 145, 151, 155, 214-217, 248

Cirillo Alessandrino 100, 106, 118, 245

Clemente XI, papa 161

Cleomede 49, 64, 72, 79, 93-94 e nn. 292-293, 95, 124, 179, 194, 196, 249-250, 252

Clostomalle, Michele nn. 48, 55

Coccino, Filoteo n. 360, 219-221, 225, 246

Cobelluzzi, Scipione, cardinale 168

Comnena, Anna nn. 214, 216

Cortasmeno, Giovanni 242

Cosma di Montserrat 166

Costanza Augusta 117

Crisoberga, Andrea 166, 248

- Massimo 166

- Teodoro 162, 166, 248

Crisolora, Manuele n. 144, 63 e n. 164, 67, 131, $147,154,172,183$

Cumno, Foca n. 79

Damila, Antonio n. 149

Devaris, Matteo 203

Dexio, Teodoro 66, 99 e nn. 320, 322, 111 e n. 375,118

Diadoco di Fotica 102, n. 344 
Diassorino, Giacomo 179

Didimo Alessandrino 245

Diofanto Alessandrino 249, 251-252

Diogeniano 86

Disypatos Galesiota, Giorgio n. 180, 227, 231-232

Doroteo di Sidone 182

Ecumenio 245

Efestione Tebano Astrologo 84, 95, 182, 233

Eliodoro Ps. 233

Enrico IV, re di Francia 214, 217, 219, 225, 227

Ermete Trismegisto 96, 182

Ermogene di Tarso 86 e n. 243, 105

Erone Alessandrino 258

Esiodo 83-84, 105, 209

Euclide 49, 64, 72, n. 270, 96, 179

Eugenio di Sicilia 151

Eugenico, Giovanni nn. 194, 196, 197

- Marco n. 194

Euripide 80, 83 e n. 227, 84, 200-201

Eusebio di Cesarea 117

Evagrio Pontico 102, n. 344

File, Manuele 170, 237

Filopono, Giovanni 86, 92 e n. 282, 191, 262

Filostrato, Flavio 238

Filoteo di Selimbria 7 e n. 41, 8, 96-97 e nn. $309,313,179$

Gabala, Manuele n. 69

Gaddi, Francesco 188

Galeno 182

Galesiota, Giorgio nn. 55, 69, 109, 236

Gemino di Rodi 263

Gennadio di Costantinopoli 245

Georges d'Armagnac 234

Giovanni, copista n. 138

Giovanni Crisostomo 59, 66, 100, 109 e n. 372, 112, n. 398, 118, 138, 140, 245

Giovanni Damasceno n. 327, 114, 138, 140, 160 , 244-245

Gregora, Niceforo 1, 3, 5-7 e n. 41, n. 43, 9 e n. 45 , n. 55, n. 61,19 e nn. $84-85,20$ e nn. $87-88,91,21$ e n. $93,26-27$ e n. 120,28 e nn. $124-125$, n. $138,54,56,59,64-66$, n. $166,71,77,80$ e nn. 209-210, 82 e nn. 219 , $222-223,88,92-93$ e n. 288,96 e n. 305 , 98-99, 103 e n. 348, n. 355,106 e n. 359 , n. $380,114,117$ e n. $397,119,124,128,132$, 135, 168, 179, 219, 222-223, 225, 227

Gregorio (Giorgio) di Cipro nn. 45, 55, 164

Gregorio Corinzio 237

Gregorio dei Mangani 236

Gregorio di Nazianzo 66, 86 e n. 243,100 , n. $334,102,104,107,118,244-245$

Gregorio di Nissa 59, 79, 100, 107, 112 e n. 385, 113-114, 119, 138, 146, 168-170, 212, 245

Gregorio XV, papa 174,178

Ignazio Diacono n. 216

Ineote, Andronico 6, 95, 124

Isacco di Ninive 102, n. 344

Isidoro di Kiev 95, 138, 187, 234

Iuliis de, Giuseppe 132

Jean de Fontibus, abate 164

Jean Hurault de Bostaillé 211-212

Lascari, Giano 183

Le Tellier, Charles Maurice 234

Leonzio di Costantinopoli 109, 111, n. 398, 140

Leone X, papa 161

Lesbonatte 209

Libanio 131, 140, 177

Lollino, Alvise (Luigi) 168, 171

Luciano di Samosata 4, 59, 79, 83, 85-86, 104-105, 112 e n. 384, 119, 174-178

Luciano Ps. 175, 177

Luigi Filippo, d’Orléans, re di Francia 233

Lullude, Michele nn. 55, 60

Magistro, Tommaso n. 215

Malace, Giovanni Duca 52 e n. 145, 56, 61, 66, $109,115,157,248$

Malaxos, Manuele n. 53

Manetti, Giannozzo 178

Martino V 166

Massimo Confessore 114, 244-245

Medici de', Caterina 217

- Lorenzo 183

Meliteniota, Teodoro . 265, 92

Mendoza de, Diego Hurtado 135

Mesarita, Giovanni n. 128

Michelozzi, Niccolò 200, 209

Minoïde Mynas 239

Mirocefalita, Michele 169, 171

Moscopulo, Manuele 85 e n. 237, 200-201, 209 
Murad I, sultano ottomano 161

Murmure, Nicola 135

Niceforo I, patriarca n. 216, 117, 245

Niceta di Maronea 119, 162-163, 166

Niceta Myrsioniotes 225

Niccolò V, papa 166

Nicola Rhabdas Smirneo 258

Nicola Cabasila 91, 149, 219-221, 223-225, 255

Nicola di Metone 108 e n. 368, 112, n. 398, 139,146

Nicomaco 93, nn. 294-295, 195-196

Nifone, monaco 66, nn. 344, 380

Origene 112, 119, 212

Orsini, Fulvio 192

Pachimere, Giorgio n. 218, 163

Paciaudi, Paolo Maria, cardinale 210

Palama, Gregorio 1 e nn 4, 6, 7, 20, 98-99, n. 344,109 e n. $371,119,143,145$

Paleologo, Giovanni V, imperatore 119

Paolo, san 114, 244

Pappo Alessandrino 91, 132, 135, 254, 256

Pediasimo Poto, Giovanni 80 e n.209, n. 293, 179,258

Petrarca n. 275

Piccolomini, Enea Silvio 151

Pico della Mirandola, Giovanni 183

Pierre de Girardin, ambasciatore francese 232

Planude, Massimo n. 55, n. 138, 80, n. 209, n. 224 , n. $352,168,170,246,251-252,258$

Platone 176-177

Plutarco 71, 114, 119, 191, 228

Polibio Sardiano 237

Poliziano, Angelo 206

Porfirio n. 213, 87, 92, 132, 134-135, 190-191

Porzio, Simone 135

Proclo Diadoco 259

Proclo Ateniese 138

Procopio di Gaza 108 e n. 368, 112, n. 398, 138-139

Provataris, Emanuele . 53

Psello nn. 211, 214, 82, nn. 217, 243, 314

Rauchfuss, Conrad n. 270

Retorio 234

Ridolfi, Niccolò, cardinale 206, 209, 217, 225
Sacchi Bartolomeo, il Platina 144, 151

Sevin, François 233

Simeone Grammatico 237

Sincello, Michele 237

Sinesio di Cirene n. 78

Sisto IV, papa 135, 144

Stefano Alessandrino 183, 261

Strozzi, Palla 212

- Piero 217

Teocrito 83-84, 209

Teodoreto 245

Teodoro Grapto 117 e nn. 397-398

Teodoro Metochita nn. 45, 85, 80 e n. 209

Teofane di Nicea 149

Teofilatto di Bulgaria 246

Teone Alessandrino 91, 92 e n. 282, 254, 256, 262

Tolomeo, Claudio 2, 4-6, 16, 20-21 e n. 92, 46, $49,64,73,80,82$ e n. 222, n. 261, 91 e n. 277, 92, 93, 95 e n. 299, 105, 112 e n. 384, 121, 132-135, 179, 187, 196, 249, 253-254, 256, 262

Tolomeo Ps. 234

Tommaso d'Aquino 63, 115

Trapezunzio, Giorgio n. 279, 256

Triakontaphyllos, Giovanni 166

Tricline, Nicola n. 55, 29, 131

Triclinio, Demetrio n. 55, 14 e nn. 60-61, 29, nn. 131, 224, 84, 201, 205

Trifone I, Grammatico 208-209, 237

Trifone II, Grammatico 237

Trivizia, Giorgio n. 149

Trivoli, Demetrio 183

Tzetze nn. 212, 213, 204

Tzicandile, Manuele n. 55, 45 e n. 140, 60, $71-72,119,160,227,232$

Urbano VIII, papa 168

Vladimír Sís 239

Zaccagni, Alessandro Lorenzo, cardinale 161 Zanetti, Anton Maria 240, 249, 253, 257

Zigabeno, Eutimio 138

Zografo, Gedeone 99, 101 e n. 335, 143

Zonara Ps. 139 
